eyepiece was used, would be only 25 in., or approximately equal in light-gathering power to a refractor of $20-i n$. aperture. A power not less than 216 would be necessary to give the full benefit of the large mirror. Although the eyepiece in question was not the only one employed, it may be important to take account of the fact that some of the observations at Parsonstown were not made with the full aperture of the telescope (Journ. B.A.A., vol. xxvi., p. 302).

\section{VENTILATION AND METABOLISM.}

$\mathrm{T}$

HE New York State Commission on Ventilation has issued an outline statement of the work done in I9I5. In the first report the Commission supported the view of the English physiologists, that the principal factors which make for comfort are temperature humidity and air movement, and that the effects of poor ventilation cannot be explained by the presence of volatile organic poisons in the air or any chemical change in the atmosphere. Even slight differences in temperature produce characteristic physiological responses in the body, affecting the output of physical work and likewise the inclination to do mental work. "In only one respect did the chemical quality of the air breathed show any characteristic effect on the body mechanism, this effect appearing in the slightly diminished appetite for food in a stale, unventilated atmosphere."

The Commission has now sought to find what quality of the stale used air has this effect. Is it the odour present? the increased $\mathrm{CO}_{2}$ ? or what? Artificial body odours and excess of $\mathrm{CO}_{2}$ have been introduced into a room ventilated with fresh air, but these have not produced the effect on the appetite. We do not believe that the Commission has ever properly eliminated the physical conditions. In their experiments they arranged that the temperature (wet and dry bulb) should be kept the same in the ventilated as in the stale-air chamber, and in the latter they placed a table fan to blow air upon the subjects, in order to imitate the current of air which circulated in the chamber ventilated with fresh air. There is no proof that the fan had this effect. It may not have ventilated the clothes of the subjects as effectually as the current of air did in the fresh-air chamber. We would suggest that the rate of cooling be measured with the katathermometer. Until this is done we cannot accept the view that the diminished appetite is due to any chemical alteration of the stale atmosphere. It seems more likely to be caused by a diminution in metabolism resulting from a lessened rate of cooling of the body surface.

The Commission says that for extreme mental concentration, involving an almost entire absence of physical exertion, a temperature of $75^{\circ}$ at $5^{\circ}$ per cent. relative humidity was preferable to $68^{\circ}$ at the same humidity, whereas for tasks involving greater motor effort, such as typewriting, the cooler temperature was coincident with the greater output. Here again data are wanted as to rate of cooling. Was the atmosphere a still one? In this country $63^{\circ} \mathrm{F}$. is regarded as a suitable temperature, but the comfort is far more a question of rate of cooling than of temperature. We would point out that mental concentration which demands an entire absence of physical exertion and so warm an atmosphere tells against the health of the body; the metabolism is greatly reduced, and with it the appetite; the digestive organs miss the massage due to bodily exercise and deep breathing; the circula tion is not made vigorous by the pumping action of the skeletal muscles and those of respiration; and the lungs are but little expanded by the shallow breathing. Daily open-air exercise is essential to compensate for NO. 244I, VOL. 97 ] such intense mental application if the health is to be maintained. Such work, together with high feeding, alcoholic pick-me-ups, and amusements taken in hot atmospheres, leads to the bodily flabbiness and middleage degeneration of the business man. The scholar requires his "constitutional" or else he will become hypochondriacal.

The Commission has examined the conditions of the nasal mucous membrane in hot and cold atmospheres, and generally confirms conclusions reached by the reviewer (cf. Lancet, May Io, I9I3). In the majority of subjects examined the reaction from heat is one of increased swelling, moisture, and redness, and the reverse from cold. Air blown upon the face by fans greatly modifies the effect. On going from the cold to the hot room with fans there is a decrease in the size of the inferior turbinates and in the amount of moisture. The characteristic change on passing from the hot to the cold condition with fans is an increase in the turbinates and secretion. The Commission reports that laundry workers show a high percentage of cases of atrophic rhinitis, the result of working in hot humid atmospheres. The changes of the nasal membrane produced by environment must materially affect the incidence of infection by "colds." This subject is dealt with by the reviewer in an article published in the British Medical Journal for April I5, I916.

Mr. Palmer, the chief of the investigating staff of the Commission, has fashioned a new sampling apparatus for the determination of aerial dust. Air is drawn, by means of an electric-driven fan, through a $\mathbf{U}$-tube containing some water. The water is thrown into a spray formation in a conical glass vessel attached to the $\boldsymbol{U}$-tube, and the air is washed of its suspended dust as it passes through the water shower. One hundred cubic feet of air can be put through in thirty minutes. The water can be evaporated and the dust weighed, or the dust can be estimated by the turbidity of the water against a set of standards, or the particles of dust-in a measured quantity of the water-counted under the microscope. The pernicious effect of dust on the lung is not properly realised by the public. Dust containing free silica is the most potent cause of phthisis prevalent in miners, granite and flint workers, etc. The motor-cars stir up clouds of dust from roads metalled with flint and granite. People dislike the dust on their clothes, but do not realise the damage it causes to their lungs. All dusts diminish the efficiency of the lungs and lead to lessened expansion and shortened breath-the asthma of dusty occupations. LEONARD Hill.

\section{THE AMERICAN PHILOSOPHICAL SOCIETY.}

THE annual meeting of the American Philosophical Society was held on April $1_{3}-\mathrm{I}_{5}$, during which nearly fifty papers were presented on a large variety of topics. The address of welcome was delivered by Dr. W. W. Keen, the president, who, with the vicepresidents, Dr. W. B. Scott and Prof. E. C. Pickering, presided at the various meetings.

We are able, from the material which the secretary, Prof. A. W. Goodspeed, has sent us from Philadelphia, to give brief abstracts of some of the papers which were read.

Dr. R. F. Bacon, "The Work of the Mellon Institute in its Relations to the Industries and to the Universities ":-

The first industrial fellowship at the Mellon Institute was founded through a grant from a baking company which desired to improve its product. The sum of money given was used, as has been all 